\title{
Technologies for mathematical and computer modeling to automate the process of operational states development for heat supply systems
}

\author{
Zoya I. Shalaginova ${ }^{1 *}$, Vyacheslav $V$. Tokarev ${ }^{1}$, Oksana A. Grebneva $^{1}$, and Aleksandr $V$. Lutsenko ${ }^{l}$ \\ ${ }^{1}$ Melentiev Energy Systems Institute of Siberian Branch of the Russian Academy of Sciences (ESI SB RAS), 130 Lermontov \\ Str., Irkutsk 664033, Russia
}

\begin{abstract}
This article proposes the new technology for development of operational states for HSS of arbitrary structure and dimension. Technology is based on multilevel modeling and a new method for adjustment calculation of thermal hydraulic states. It is implemented in the information and computer complex «ANGARA-HN». Technology includes checking the permissibility of states, calculating the throttling devices on the network and inputs of consumers' buildings. It allows calculating large systems with intermediate stages of regulation, developing adjustment measures to improve the quality of heat supply and consumer provision, reducing circulation flow rates and pressure in networks. The development of modeling methods is carried out in the following directions: taking into account the new composition of equipment, including mixing pumping stations; development of nontraditional methods of calculation, such as object-oriented modeling; development of tasks of hierarchical optimization and identification of state parameters, as task of rising for model adequacy; development of task for finding of sectioning variants for multi-circuit heat network with several sources. The application of methodological and software developments makes it possible to obtain both an economic and a social effect by identifying and realizing of the energy saving potential, improving the quality and reliability.
\end{abstract}

\section{Introduction}

Russian heat supply systems (HSS) are unique in their scale and complexity engineering structures. They unite many different types' elements that are developed over time and are dispersed over a large territory. The presence in the systems of heat sources (HS) of various types (CHP, boilers), pumping stations (PS), central or individual heat substations, many different consumers, including subsystems for heating, ventilation and hot water supply, lengthy main (MHN) and distribution heating networks (DHN), operating under constantly changing conditions, determine the complexity of the tasks of organizing and controlling of states.

The tasks of calculating of the thermal hydraulic states of HSS, the permissibility and optimization of the states are basic for the analysis and quantitative substantiation of decisions on organization of HSS operational states. At the same time, methodological, algorithmic and software of these tasks have to satisfy the following requirements: 1) adequacy to real physical processes and properties of the initial information; 2) reliability, which guarantees of solutions with predetermined accuracy; 3) high-performance; 4) the ability to solve problems of large dimension; 5) universality and adaptability with respect to the arbitrary structure of the calculation object, the laws of the medium flow, changes in the statements of calculation problems and design conditions. These requirements are dictated by: increasing complexity and dimension of HSS; introduction of new equipment; limited decisionmaking time based on calculations in dispatch control; the need of multivariate calculations in solving design and operation problems; the use of models and algorithms for flow distribution calculating in solving of other more complex problems (optimal synthesis, reconstruction, states control, identification, etc.). Automation of decision-making processes for the organization of HSS operational states is of fundamental importance, since the choice of methods for organizing of the states, the quality and optimality of decisions made in practice, depends on the experience and qualification of the engineer by state and the complexity of the calculation object.

\section{Overview of the methodological base and characteristics of software for the development of HSS operational states}

Currently, there are many methods for calculating both the hydraulic [1-5] and thermal hydraulic [6-13] states of the HSS. At the ESI SB RAS on the basis of the formulated and scientific direction - the theory of hydraulic circuits (THC), it has accumulated unique experience in creating methodological and software solutions for solving problems of calculation and

* Corresponding author: shalaginova@isem.irk.ru 
optimization of HSS [5,8-13, etc.]. To date, developed system of mathematical models and methods has been created for calculating and analyzing of the thermal hydraulic states of the HSS [11-20]. A new technique and a set of high-speed algorithms have been developed for calculating of HSS states with an arbitrary number and placement of automatic control devices [10]. The methodology for adjustment calculation of thermal hydraulic state for the organization of HSS operational states includes checking the permissibility of states, calculating throttling devices on the network and inputs to the buildings of consumers, taking into account the differentiated amendments to flow rates for compensating of heat losses in the network. The technique allows developing adjustment measures to improve the quality of heat supply and consumer provision; reduce circulation flow rates and reduce network pressure.

In the ESI SB RAS, the informational and computer complex (ICC) «ANGARA-HN» [21] has been developing for many years to automate the analysis and decision-making processes in the design, operation, and dispatch control for systems of an arbitrary structure and dimension. In the framework of the ICC, the technology for the development of operational states of large HSS with intermediate control stages [20] was implemented. It is based on multilevel modeling [8] and the technique of adjustment calculation of thermal hydraulic state [10].

The priority directions for the development of modeling methods and software are: accounting for the new composition of equipment [22] development of nontraditional calculation methods, such as object-oriented modeling [16,17]; problems of optimization [23] and identification [24], as well as adequacy of the HSS model to the real state; automation of the processes of analysis and development of operational states [25].

\section{Technology for the development and organization of large HSS states based on multilevel modeling methods}

The technology for the development of operational states of large HSS is based on multilevel modeling $[8,12,20]$. The proposed approach provides the possibility of a practical solution to the problem of quantitative substantiation of decisions on the organization of states for HSS of arbitrary dimension and structure, including HSS with intermediate control levels. It allows overcoming the contradictions between the high dimension of the problem and the requirements of the integrity and visibility of the object, applying the technology of parallel computing, which significantly reduces the calculation time. The approach is based on the methods of equivalenting and decomposition of design schemas and tasks and involves multilevel data organization and organization of single and multilevel calculations. The methodological base for highperformance multilevel adjustment calculations of thermal hydraulic states takes into account all the requirements for their permissibility $[10,12,19]$.

The process of state development using this implemented in ICC «ANGARA-HN» technology (Fig. 1) can be divided into the following stages: 1) development of a multilevel computer model of HSS, including MHN and DHN design schemas, information on the types and parameters of its elements, as well as restrictions on the state parameters; 2) analysis of the initial information; 3) analysis of current state and network bandwidth with given parameters of HS and central heat substation (CHS); 4) analysis of state violations; 5) development of measures for organizing an permissible state (entering of the state into an permissible area).

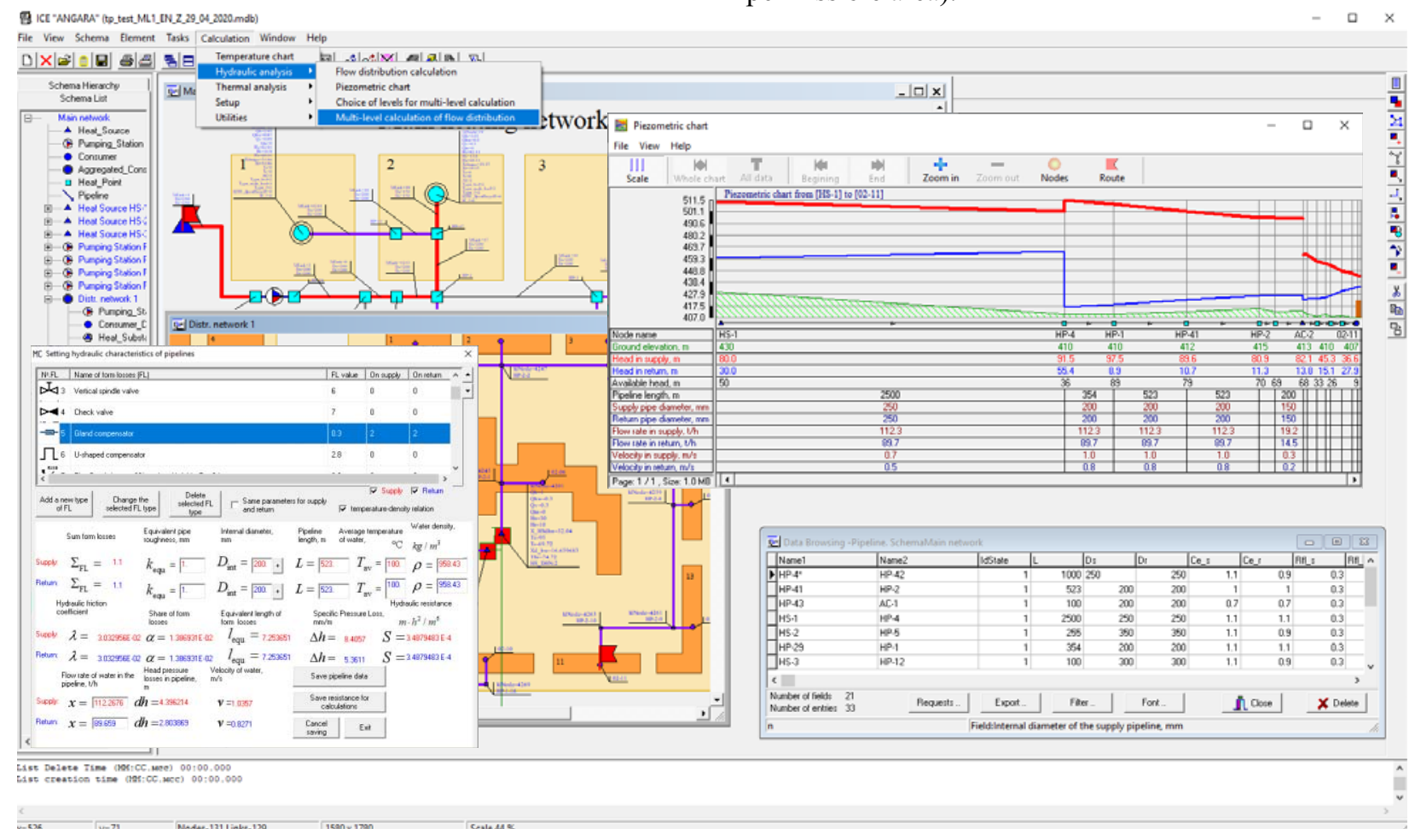

Fig. 1. Results of multilevel hydraulic calculation. 
Based on the analysis of the current state of HSS under current operating conditions, «bottlenecks», state violations are identified and a quantitative assessment of the current level of consumer supply is made. The analysis of the current state, as well as the development of controls, is based on calculations of hydraulic and thermal states. Typically, such calculations are divided into adjustment and checking. The main purpose of the adjustment calculation is to find such controls that will allow the run of given water flow rates with current water temperature through the HSS customer systems, subject to technological restrictions on the water parameters - pressure at all nodes of the system, the pressure at the consumer inlets and the water velocity.

When analyzing the state of heating networks, temperature state are important for accounting: cooling of the water along the transportation path; shortage of thermal energy among consumers; selection of measures to compensate for heat losses in the network (increase in water flow rate or entering an amendment to the temperature chart). The result of the adjustment calculation of the heating network is a state in which given heat loads of all consumers and other restrictions are provided. Restrictions arise from the requirements of permissible operating conditions of the equipment.

Entering the state into an permissible area is a difficult task and requires the use of multivariate or optimization calculations by technological and economic criteria. In practice, it is not always possible to create the permissible and even more optimal state only by regulators. In some cases, it may be necessary to reconstruct the whole system or its elements.

Checking calculations of operational states are carried out in order to determine deviations of the state parameters from the required values in non-design conditions, including the degree of consumers' provision.

The application of the developed technology in practice made it possible to identify a great potential for energy saving and significantly improve the quality of heat supply in many cities [26].

\section{Development of methods for mathematical modeling of HSS states to ensure their adequacy and optimality}

\subsection{States optimization}

Recently, the problems of energy efficiency have become increasingly relevant, and HSS have significant reserves of energy saving [27], which are caused by the nonoptimality of their operating states. Automation of solving of the problem of HSS states optimization is complicated by a number of factors: the large dimension of HSS [28], the nonlinearity of the involved flow distribution models, the need to take into account several objective functions, the presence of discrete variables of different types, etc. Therefore, there are no methods and software systems suitable for optimization of states for
HSS of real dimension. This determines the relevance of developing methods for optimizing of HSS states.

During optimization, it is considered that temperature charts for $\mathrm{HS}$ are set, heat losses in networks are eliminated, and their residual value can be neglected. At the same time, the requirements for a sufficient supply of thermal energy to consumers are reduced to the need to maintain the required heat carrier flow rates for consumers, and the task is to optimize the hydraulic state. The case of parallel operation of pumps of the same type at the PS is considered. This case is typical for HSS.

Substantially, the task of optimizing of the HSS hydraulic state is to find control actions that ensure the implementation of state that corresponds to the permissibility requirements and the specified optimization goals. Energy saving requirements can be reduced to minimizing a single economic objective function, which is used as a variable component of the costs for maintaining of the state [29]. The desire to minimize the complexity of adjustment measure and reduce possible water leaks and the risks of emergency situations can be reduced to minimizing additional places of flow control and minimizing the total pressure in the network.

To overcome the dimension of problem, to separate different types of discrete variables and objective functions by different tasks, a hierarchical approach to optimizing of the HSS hydraulic state was proposed [30]. The method consists of the following steps: 1) decomposition of HSS into MHN and DHN; 2) the search for the limits of permissible changes of the state parameters in the decomposition point; 3) optimization of MHN hydraulic state, taking into account the restrictions obtained in the previous step; 4) optimization of DHN hydraulic state taking into account MHN hydraulic state. The MHN level includes all HSs, PSs, and the multi-circuit part of the network. The DHN level includes branched passive networks to end consumers. The decomposition point is the point of connection of DHN to MHN in a single-linear representation and two nodes in the bilinear. One of these nodes is the junction of the supply pipelines of MHN and DHN, the second return. For $\mathrm{MHN}$, the decomposition point is an aggregated consumer with a given water flow rate and two-sided restrictions on the pressures in the supply and return pipes, as well as the difference in these pressures. For DHN, the decomposition point is aggregated HS.

The search for the limits of permissible changes in the state parameters at the decomposition point is as follows. The water flow rates at the decomposition point are easily found based on the flow rates of consumers and nodal flow rates in the DHN [30]. The search for minimums and maximums of pressures in the supply and return pipelines, as well as the pressure difference between them, is carried out by solving of six optimization problems [30].

As a rule, HSSs are designed in such a way that no additional controls are required when optimizing of hydraulic state of MHN. This task is to minimize the economic objective function. For this, a triple nested iteration loop is used. On the internal cycle, the

* Corresponding author: shalaginova@isem.irk.ru 
permissible hydraulic state of MHN is searched, on the average, the minimum of the economic objective function with by the continuous variables, and on the external, the discrete variables are selected that are responsible for the number of turned pumps on PS by the continuous method of branches and boundaries [29].

When optimizing the hydraulic state of DHN, the number of additional flow control points and the overall pressure level in the network are minimized. To solve this problem, an independent dynamic programming method has been developed with circuit equivalents [31], based on the Bellman optimality principle [32] and the ability to reduce dimension of DHN design schema to one branch by unification of parallel and sequential branches.

The hierarchical approach to optimization of HSS hydraulic state presented in the report has the following advantages: 1) the possibility of optimization hydraulic state for HSS of real dimension; 2) the possibility to simultaneously use several objective functions that correspond to real practical problems; 3) high performance and accuracy compared to the approaches of other teams of authors.

\subsection{Simulation of mixing pumping stations}

HSS schemas in large cities include control elements such as mixing PS. These elements are used to lower the temperature in the supply pipeline by mixing of water from the return pipeline. This allows realizing a transition to a lower temperature chart of quality control. Mixing PS is common in HSS, which have many compactly located consumers with low heat load and direct connection. In this case, to increase throughput capacity, the MHN from the HS to the mixing PS can operate according to an increased temperature chart.

In a number of cities, a multistage cascade change in temperature chart is used. For example, the heat carrier is supplied from the source in transit according to the schedule $170 / 70^{\circ} \mathrm{C}$ to the mixing PS located at the entrance to the city. Then, with the help of cascade of mixing PSs, the temperature chart is lowered to $150 / 70^{\circ} \mathrm{C}, 130 / 70^{\circ} \mathrm{C}$ and ultimately in front of the group of directly connected consumers to $95 / 70^{\circ} \mathrm{C}$.

Since, the water flow rate through the mixing PS depends on the temperature of the mixed water, and it, in turn, depends on the flow distribution and heat losses in the network. It is not possible to determine the flow distribution in one calculation of hydraulic and thermal condition, an external iteration cycle is required, in which the stop criterion will be achievement of the required temperature of mixed water with a given accuracy. In addition, to calculate the temperature distributhin in HSS, it is necessary to apply special methods and stopping criteria. This is because closed flow circuits appear in the network. In the case of cascade mixing, these circuits are embedded [33].

At the nodal temperatures fixed at the $k$-th iteration of the external cycle, the hydraulic state are calculated. In this state, the flow rate of the mixed water through the mixing PS is uniquely determined by the flow rate of the network water in the supply pipeline before mixing PS and the mixing coefficient.

Thus, when calculating of the hydraulic state, a pipeline with mixing PS can be considered as a pipeline with an active pressure. As well as this pipeline is a flows ratio controller (FRC) by the temperature of mixed water.

The statement of the problem of adjustment calculation of hydraulic state is as follows. Given: design schema; hydraulic characteristics for all its branches; nodal flow rates in $(m-1)$ nodes; vector of known pressure increments for active elements (acting pressures of HSs, PSs and mixing PSs); mixing ratios for the mixing PS; pressure in one of the nodes. It is required to determine the flow rates and pressure drops across all branches, as well as nodal pressures in $(m-1)$ nodes that satisfy the operational states of the mixing PS.

When the adjustment calculation of the thermal hydraulic calculation is carried out, the mixing unit (with the mixing PS) is modeled by a temperature controller. This controller has fixed temperature at the mixing PS outlet. In this case, the task of the adjustment calculation of thermal hydraulic state is joined to determining the flow distribution in the network to ensure the required loads of consumers, including the flow rate of water mixed from the return pipeline.

The proposed methodology is based on a combination of the following calculation stages [22]: 1) decomposition of the calculation of the thermal hydraulic state into hydraulic and thermal state calculations; 2) the use of fictitious FRC with a given mixing ratio of flows at the mixing PS output when calculating of hydraulic state; 3) fixing of the temperature at the outlet of the mixing PS in the calculation of thermal state; 4) correction of the FRC setting according to the results of the thermal state calculation; 5) iterative implementation of p. 2-4 until the stabilization of the mixed water flow rate with a given accuracy.

The proposed approach is implemented in a modified module for calculating the flow distribution using the relay method for calculating of the hydraulic circuit with controlled parameters. It allows determining the thermal hydraulic state of HSS with the mixing PS without using decomposition design schema of the network, which requires a laborious coordination of the boundary parameters in the points of decomposition.

\subsection{Sectioning of multi-circuit heating networks}

HSS of large cities, as a rule, have a multi-circuit structure. Valves on the multi-circuit network, control elements, changing the parameters of HSs and PSs allow the redistribution of flows. Due to redundancy, HSS of multi-circuit structure has greater reliability then the radial structure. However, in normal states the HSS are preferred to operate by a branched (radial) schema, without heat transfer flows between the heat mains. Each main has a unique composition of connected consumers. 
Such a scheme simplifies the processes of network adjustment, detection and localization of accident sites.

To convert the operation schema of a multi-circuit heating network from a multi-circuit to a radial one, it is necessary to determine the places for cutting the circuits (sectioning of the schema), while minimizing the increase in energy costs to create a hydraulic state for the HSS.

In ICC «ANGARA-HN», the task of finding a sectioning variant for multi-circuit heating network with several HSs is formalized [33]. The task of sectioning is posed as optimization. The search for promising sectioning involves multivariate calculations of thermal hydraulic state. This solves the problem of finding the permissible state. The main criteria in the task of HSS sectioning are technological criteria. As such a criterion can use the value of hydraulic power expended on circulation in HSS. This value is definitely determined through the hydraulic power of HS.

The loss of hydraulic power at fixed flow rates, determined from the loads of consumers. Hydraulic power can be divided into two parts - the required and the excess. The total value of the required hydraulic power for consumers is constant, and for each consumer depends on the required differential pressure (available pressure) at the inlet to the consumer and the design flow rate of network water. The excess pressure drop at HS is equal to the excess pressure drop of the consumer dictating by this parameter. The product of the excess differential pressure of the network water and its flow rate is the excess hydraulic power of HS. The task is to find a sectioning variant in which the sum of excess hydraulic powers of all HSs for HSS will be maximum.

The methodology for solving of the problem of searching for rational sectioning of HSS consists in the sequential implementation of the following points: drawing up and calculating of HSS schema without sectioning; search for a sectioning variant near the nodes of the flows gathering; redistribution of flows between HSs; redistribution of flows within independent fragments of network.

Various sequential of variant search for sectioning have been investigated, based on network sectioning close to flows gathering nodes. To automate the solution of the problem of searching for rational sectioning, it was necessary to solve and implement the following subtasks as part of ICC «ANGARA-HN»: calculation of the flow distribution for HSS schema without sectioning; search for flows gathering nodes and ranking of branches included in these nodes by flow rate; calculation of the minimum required available pressure for aggregated consumers; search for dictating consumers according to various criteria; implementation of a scheme of iterative calculations of hydraulic state to determine rational sectioning.

When testing the methodology with a real example, it was possible to find a sectioning variant that reduces the total loss of generated hydraulic power at CHP by $3 \%$, compared with the variant obtained by the method of simultaneous network sectioning at the points of flow gathering.

\subsection{Ensuring the adequacy of HSS models}

Not knowing the true values of the actual characteristics and parameters is the main deterrent to the effective application of mathematical and computer modeling methods to ensure the adequacy of HSS models to their real state.

In practice, this problem is solved by conducting special active tests. However, this does not alleviate the problem described above due to the poor regulation of the conditions for the testing used in tests methods, as well as the lack of a guarantee for obtaining of the results of the required completeness and accuracy.

The tasks of determining the HSS characteristics coefficients from the measurement results are tasks of parametric identification. To solve such problems, methods are potentially applicable [34-39]. However, their use in conditions of passive observations of the normal functioning of HSS does not guarantee an optimal solution due to the lack of used measuring devices and the small range of variation of the states involved for identification.

To develop HSS operational states, at the first stage, it is proposed to check the adequacy of the mathematical models, using the energy systems developed and developed at ISEM SB RAS methods of the active identification for HSS [40-43]. The technique is a sequential (step-by-step) planning strategy when the next experiment is planned taking into account the information obtained after processing the results of the previous experiment.

Vector of state parameters $\mathbf{R}$ consists of vectors of independent $\mathbf{X}$ and dependent $\mathbf{Y}$ parameters of model. Hence, the model in general can be written as $U(\mathbf{Z})=U(\mathbf{R}, \boldsymbol{\alpha})=U(\mathbf{X}, \mathbf{Y}, \boldsymbol{\alpha})=0$. So vector of dependent parameters is $\mathbf{Y}=F(\mathbf{X}, \boldsymbol{\alpha})$, where $F$-is an implicit function. The determinant of the covariance matrix of element parameters $\left(\operatorname{det} \mathbf{C}_{\boldsymbol{\alpha}}\right)$ acts as an optimality criterion. This criteria is the function of independent state parameters $\mathbf{X}$, elements parameters $\boldsymbol{\alpha}$, and as well as it depends on measurement devices [34]. Each step of active identification technique (Fig. 2) includes solving of following tasks: 1) state planning [42]; 2) measurement devices placement [43]; 3) test; 4) processing of test results [34].

As an additional criterion for assessment the adequacy of the model, it is proposed to use the criterion of minimizing the maximum response variance $\gamma=\max _{j} \frac{\hat{\sigma}_{j}^{2}}{\tilde{\sigma}_{j}^{2}}$. The value of the proposed criterion shows how many times the variance of the estimate of an unmeasured parameter $\hat{\sigma}_{j}^{2}$ more variance of its direct measurement $\tilde{\sigma}_{j}^{2}$. 


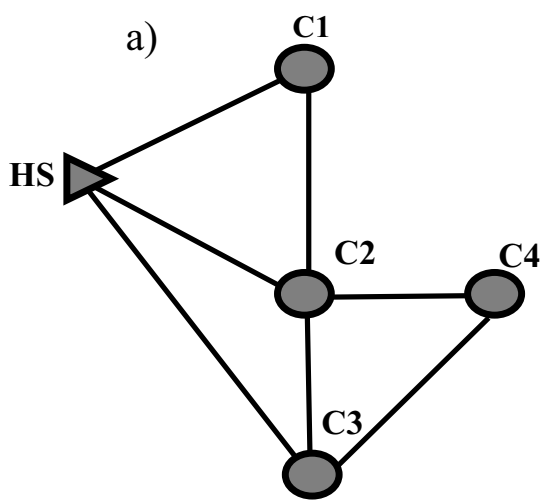

c)

$\lg (\mathrm{D})$

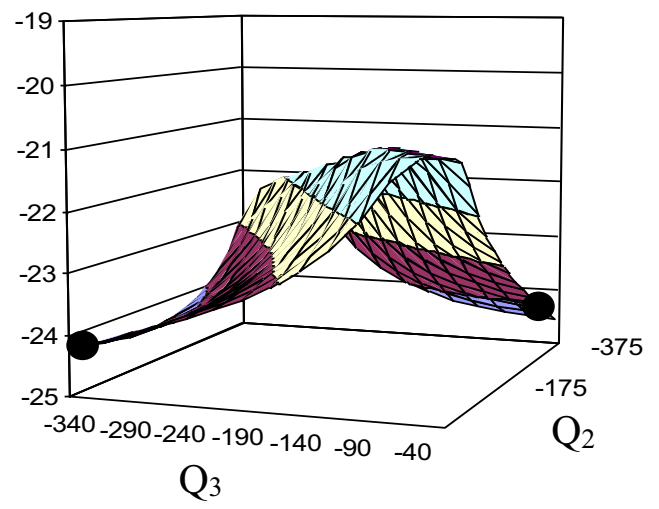

b)

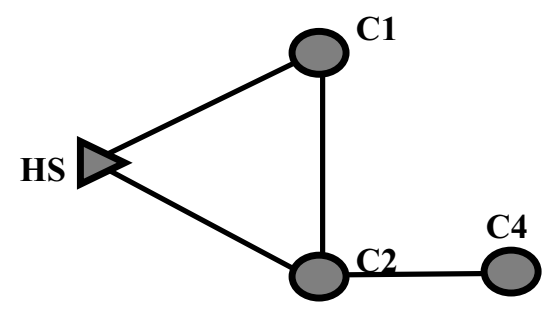

d)

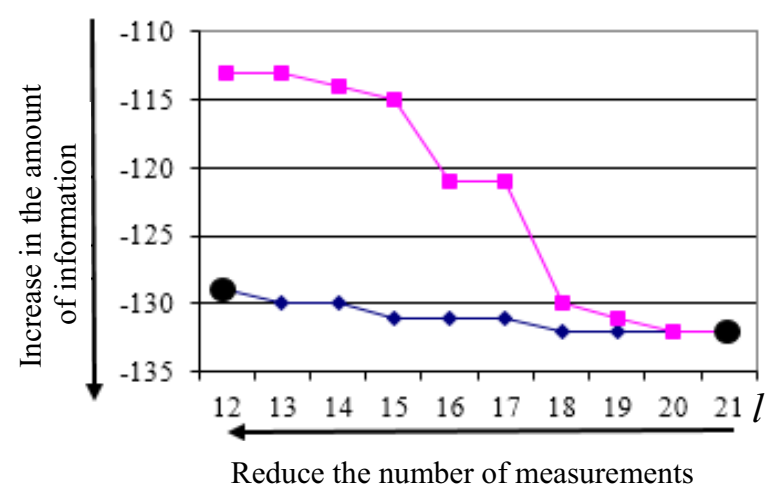

Fig. 2. Illustration of the methodology for active identification of HSS: a) the initial scheme of HSS; b) a fragment of HSS for testing; c) an illustration of the multicriteria of the task of state planning; d) illustration of the search for the optimal composition of measurement devices ( $\mathrm{C}$ is the consumer; $\mathrm{D}$ is the information criterion; $\mathrm{Q}$ is nodal flow rate; $l$ is the number of measurement devices).

When solving of the task, the obtained values of the additional criterion are compared with the necessary (required) accuracy of the predictive properties of the model, i.e., its adequacy (Fig. 3).

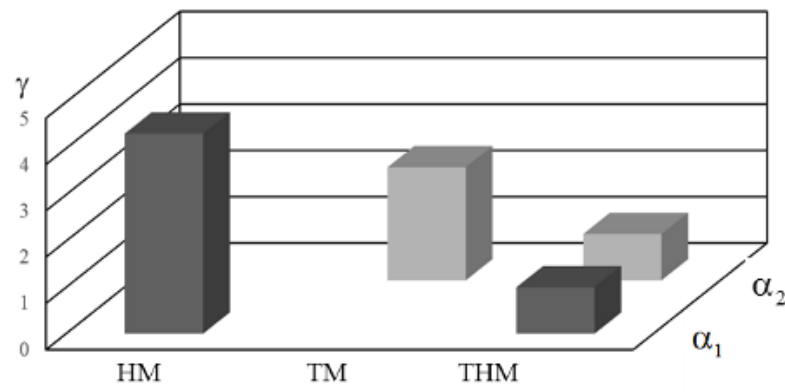

Fig. 3. Diagram of prediction errors by types of used models (HM - hydraulic model; TM - thermal model; THM - thermal hydraulic model; $\alpha$-elements parameters, index 1 for hydraulic state, index 2 for thermal state).

The effectiveness of the proposed method consists in minimizing the number of experiments to obtain the required or the maximum achievable in accuracy predictive properties of HSS model.

The proposed technology for identifying of HSS with an appropriate level of software implementation allows providing a qualitatively new level of model adequacy and the effectiveness of their application in solving various problems.

\section{$5 \quad$ Practical use}

The application of the abovementioned methodological and software developments allows obtaining both economic and social effects by identifying and realizing the potential of energy and resource conservation in organizing the operation states of HSS, improving the quality and reliability for supply of the population and industry with thermal energy. The development results have found effective application in the design, dispatch, optimization of HSS states in various enterprises $[26,44,45]$. Using the proposed methodological apparatus, calculations were made on the development of states and adjustment measures in many Russian and foreign cities, such as Irkutsk, PetropavlovskKamchatsky, Taishet, Angarsk, Bratsk, Baikalsk, Cheremkhovo, Zheleznogorsk-Ilimsky, Ust-Kut, St. Petersburg , Ulan Bator (Mongolia), Darkhan (Mongolia), Dnepropetrovsk (Ukraine) and many others. The implementation of the planned measures in these cities made it possible without significant capital investments in the reconstruction of networks to normalize the heat supply to consumers; significantly improve its quality; reduce circulation flow rates; reduce 
the risks of emergencies due to compliance with the requirements of technological permissibility of states and obtain a significant energy saving effect.

The studies were carried out in the framework of project III.17.4.3 of the basic research program of the SB RAS (AAAAA17-117030310437-4).

\section{Reference}

1. E. Todini , S. Pilati , Comp. Appl. in Water Supply, 1, 1 (1988)

2. M. Vesterlund, J. Dahl, En. Conv. and Management, 89, 555 (2015)

3. E. Guelpa, C. Toro, A. Sciacovelli, R. Melli, E. Sciubba, V. Verda, Energy, 102, 586 (2016)

4. G. Schweiger, P.-O. Larsson, F. Magnusson, P. Lauenburg, S. Velut, Energy. 137, 566 (2017)

5. A.P. Merenkov, V.Ya. Hasilev, Theory of hydraulic circuits (Nauka, Moscow, 1985)

6. W. Jinda, Zh. Zhigang, Zh. Jianing, Energy Conversion and Management, 120, 294 (2016)

7. M. Kuosa, K. Kontu, T. Mäkilä, et al., Applied Thermal Engineering, 54(2), 450 (2013)

8. N.N. Novitsky, A.V. Alekseev, et al. Energy, 84, 151 (2019)

9. N.N. Novitsky, et al. Energy Industry Development and Ecology, 145 (Mongolia, 2010)

10. V.V. Tokarev, Z.I. Shalaginova, Thermal Engineering, 63(1), 68 (2016)

11.Z.I. Shalaginova Matec web of conferences, 212 (2018)

12.Z.I. Shalaginova, V.V. Tokarev, E3S Web of Conferences, 39 (2018)

13. Z.I. Shalaginova, V.V. Tokarev, E3S Web of Conferences, 39 (2018)

14. A.V. Alekseev, et al., Mathematical models and methods for evaluating and realizing the energy supply potential in the management of heat supply systems, 38 (Nauka, Novosibirsk, 2009)

15.Z.I. Shalaginova, Thermal Engineering, 63(3), 222 (2016)

16. N.N. Novitsky, E.A. Mikhailovsky, Bulletin of ISTU, 7, 170 (2012)

17. N.N. Novitsky, E.A. Mikhailovsky, Bulletin of ISTU, 21(9), 157 (2017)

18. Z.I. Shalaginova, Thermal Engineering, 51(7), 554 (2004)

19. N.N. Novitsky, A.V. Alekseev, Pipeline energy systems. Development of Theory and methods of mathematical modeling and optimization, 228 (Nauka, Novosibirsk, 2008)

20. N.N. Novitsky, et al., Proceedings of the RAS. Energetics, 1, 12 (2018)

21. N.N. Novitsky, et al., Bulletin of ISTU, 22(11), 126 (2018)
22. V. Tokarev, N. Novitsky, MATEC Web of Conferences, 212, 02006 (2018)

23. A.V. Lucenko, E3S Web of Conferences (2018)

24. N.N. Novitskii, O.A. Grebneva, V.V. Tokarev, Thermal Engineering, 65 (7), 453 (2018)

25. N.N. Novitsky, A.V. Alekseev, University News. Investments. Building. Realty, 8(4), 139 (2018)

26.Z.I. Shalaginova, V.V. Tokarev, Thermal Engineering, 66(10), 714 (2019)

27. V. Roshchanka, M. Evans. Playing Hot and Cold: How Can Russian Heat Policy Find Its Way Toward Energy Efficiency? (National Technical Information Service, USA, 2010)

28. S. Hodgson, District heating and CHP in Russia: Room for improvement, Cogeneration and On-site Power Production (2009)

29. N.N. Novitsky, A.V. Lutsenko, J. Global Optim, 66 (1), 83 (2016)

30. A.V. Lutsenko, Proceedings of young scientists at ESI SB RAS, 45, 21 (2015)

31. A.V. Lucenko, E3S Web of Conferences, 03003 (2018)

32. R. Bellman, Dynamic Programming (Publishing House of Foreign Lit, Moscow, 1960)

33. V.V. Tokarev, Thermal Engineering, 65(6), 400 (2018)

34. N.N. Novitsky, Estimation of hydraulic circuit parameters (Nauka, Novosibirsk, 1998)

35. S. Lingireddy, L. E. Ormsbee, Civil Engineerng and environmental Systems, 19(1), 13 (2012)

36. S. Alvisi, E. Creaco, M. Franchini, Urban Water Journal, 8 (4), 203 (2011)

37. Z.Y. Wu, Th. Walski, R. Mankowski, et al, $A W W A$ IMTech Conference, 1 (2002)

38. G. Yu, R.S. Powell, International Journal of Systems Science, 25(12), 2155 (1994)

39. R.I. Ogie, N. Shukla, F. Sedlar, T. Holderness, Sustainable Cities and Society, 35, 385 (2017)

40. O.A. Grebneva, Problems research and algorithms development for conditions of active pipeline systems identification: the author's abstract. Diss ... Cand. Tech. Sciences (ESI SB RAS, Irkutsk, 2005)

41. O.A. Grebneva, N.N. Novitsky, Pipeline Energy Systems: Methodological and Applied Problems of Mathematical Modeling, 220 (Nauka, Novosibirsk, 2015)

42. O.A. Grebneva, System Research in Energy, 31, 48 (ISEM SB RAS, Irkutsk, 2001

43. O.A. Grebneva, N.N. Novitsky, Thermal Engineering, 9, 70 (2014)

44.Z.I. Shalaginova, Thermal Engineering, 11, 62 (2014)

45. V.V. Tokarev, Z.I. Shalaginova, Bulletin of ISTU, 59(12), 240(2011) 